\section{Novel Enantiomerically Pure Ferrocenes}

Adolphus A. H. van der Zeijden*,a, J. Sieler ${ }^{\mathrm{b}}$, E. Hovestreydt ${ }^{\mathrm{c}}$

a Institut für Anorganische Chemie, Martin-Luther-Universität Halle-Wittenberg, Geusaer Strasse, D-06217 Merseburg

b Institut für Anorganische Chemie, Universität Leipzig, Linnéstrasse 3, D-04103 Leipzig

c Siemens, Analytical X-ray Systems, D-76181 Karlsruhe

Z. Naturforsch. 51b, 437-440 (1996); received July 26, 1995

Chiral Cyclopentadienyl Ligand, Nitrogen-Functionalized Cyclopentadienyl Ligand, Ferrocene, Ammonium Salt, Crystal Structure

A chiral ferrocene $\left(\mathrm{Cp}^{\mathrm{E}}{ }_{2} \mathrm{Fe}\right)$ was synthesized by reaction of $\mathrm{Cp}^{\mathrm{E}} \mathrm{Li}$ with $\mathrm{FeCl}_{2}(\mathrm{THF})_{1.5}$ The cyclopentadienyl ligand $\mathrm{Cp}^{\mathrm{E}}$ contains a dimethylamino group that is separated from the $\mathrm{Cp}$ backbone by two stereogenic carbon centres. Reaction of $\mathrm{Cp}^{\mathrm{E}}{ }_{2} \mathrm{Fe}$ with excess $\mathrm{MeI}$ affords the ammonium salt $\mathrm{Cp}_{2}{ }_{2} \mathrm{Fe} .2 \mathrm{MeI}$. Its solid state structure was determined by X-ray diffraction methods.

There is a growing interest in cyclopentadienyl (Cp) ligands that possess a functional donor group capable of intramolecular coordination [1]. Meanwhile, the easily accessible [2-(dimethylamino)ethyl]cyclopentadienyl ligand has found extensive use in (transition) metal chemistry [2]. Recently, we reported on the design of an enantiomerically pure analogue $\left(\mathrm{Cp}^{\mathrm{E}} \mathrm{H}\right)$ characterized by the presence of two stereogenic carbon centres between the $\mathrm{Cp}$ and the dimethylamino groups [3]. It can be expected that such ligands find increased application in transition metal promoted asymmetric syntheses and catalyses. In this paper we report on the synthesis of the first transition metal complexes bearing the $\mathrm{Cp}^{\mathrm{E}}$ ligand, as well as the absolute configuration of one of them as determined by $\mathrm{X}$-ray diffraction.

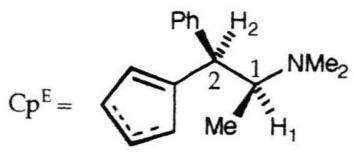

* Reprint requests to Dr. A. A. H. van der Zeijden.
The ferrocene derivative $\mathrm{Cp}_{2}{ }_{2} \mathrm{Fe}$ was prepared in $60 \%$ yield by reaction of $\mathrm{Cp}^{\mathrm{E}} \mathrm{Li}$ and $\mathrm{FeCl}_{2}(\mathrm{THF})_{1.5}$. The air-stable orange compound is very soluble in all common organic solvents, and all attempts to obtain crystals of this compound suitable for X-ray diffraction work were unsuccessful. We therefore decided to prepare a saltlike derivative by the reaction of $\mathrm{Cp}^{\mathrm{E}}{ }_{2} \mathrm{Fe}$ with $\mathrm{MeI}$ affording the ammonium iodide $\mathrm{Cp}^{\mathrm{E}}{ }_{2} \mathrm{Fe} .2 \mathrm{MeI}$. This compound indeed proved to have better crystallization properties, and its solid state structure was determined by X-ray diffraction methods.

The compound crystallizes in the non-centrosymmetric space group P1. The absolute configuration of its structure ( $97 \%$ probability) was determined by anomalous X-ray diffraction methods. The stereogenic centres $C_{1}$ and $C_{2}$ both possess the $\mathrm{S}$ configuration, as has already been suggested by NMR data [3]. Three doubly charged ferrocene cations and six iodide anions make up for the unit cell. Despite the unusual number of $\mathrm{Z}=3$, the three independent ferrocene molecules are strikingly similar. The molecular structure of one of them is depicted in Fig. 1. Selected bond distances and angles are given in Table I. Each molecule possesses a non-crystallographic $C_{2}$ symmetry axis which is running through the iron centre and is pointing to the middle of the lines connecting the equivalent atoms on the two substituents. The two substituents on the $\mathrm{Cp}$ rings show a rotation of about $83^{\circ}$ away from an eclipsed conformation. Other 1,1'-disubstituted ferrocenes show angles in the entire range of 0 to $180^{\circ}$, so that these values seem to be more guided by crystal packing forces than by intramolecular repulsion [4]. On the other hand, it is striking that, despite this rotational freedom, all three independent molecules have the same $83^{\circ}$ angle.

The major, but still small, difference between the three molecules is presented by the arrangement of the substituents around $\mathrm{C}(2)$ (the carbon atom bound to the $\mathrm{Cp}$ ring). The $\mathrm{C}(2)-\mathrm{C}(\mathrm{Ph})$ bond lies more or less in the plane of the $\mathrm{Cp}$ ring with declinations between 8 and $20^{\circ}$, whereas the $\mathrm{C}(2)$ $\mathrm{C}(1)$ bond is almost perpendicular to it (deviation: $\left.71-79^{\circ}\right)$. There is a small distortion of the tetrahedral symmetry around $\mathrm{C}(2)$ : the $\mathrm{C}(1)-\mathrm{C}(2)-\mathrm{C}(\mathrm{Ph})$ angle is opened up to about $117-119^{\circ}$ at the expense of the $\mathrm{C}(1)-\mathrm{C}(2)-\mathrm{C}(\mathrm{Cp})$ angle $\left(105-108^{\circ}\right)$. In contrast, the conformations around the $\mathrm{C}(1)$ $\mathrm{C}(2)$ and $\mathrm{C}(1)-\mathrm{N}$ bonds in all six independent substituents are identical within error limits (a Newman projection is shown below). The hydrogens atoms $\mathrm{H}(1)$ and $\mathrm{H}(2)$ are in a gauche position. 


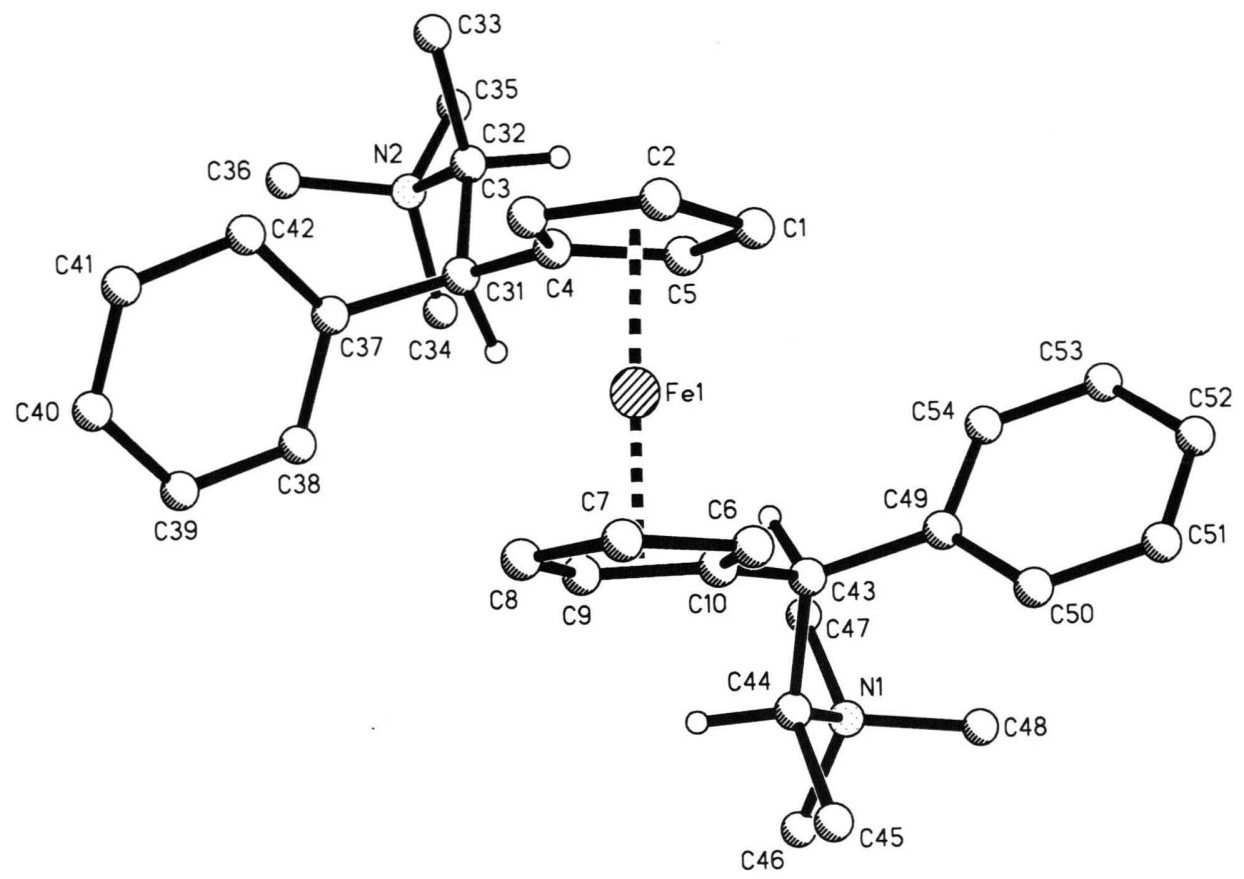

Fig. 1. Molecular structure of $\mathrm{Cp}^{\mathrm{E}}{ }_{2} \mathrm{Fe} .2 \mathrm{MeI}$ (molecule 1 shown). All hydrogen atoms, except those on the carbon atoms connecting the $\mathrm{Cp}$ ring with the nitrogen atom, were omitted.

\begin{tabular}{llllll}
\hline $\mathrm{Fe}(1)-\mathrm{C}(1)$ & $2.03(2)$ & $\mathrm{Fe}(1)-\mathrm{C}(2)$ & $2.05(2)$ & $\mathrm{Fe}(1)-\mathrm{C}(3)$ & $2.05(2)$ \\
$\mathrm{Fe}(1)-\mathrm{C}(4)$ & $2.055(13)$ & $\mathrm{Fe}(1)-\mathrm{C}(5)$ & $2.031(14)$ & $\mathrm{Fe}(1)-\mathrm{C}(6)$ & $2.049(10)$ \\
$\mathrm{Fe}(1)-\mathrm{C}(7)$ & $2.05(2)$ & $\mathrm{Fe}(1)-\mathrm{C}(8)$ & $2.06(2)$ & $\mathrm{Fe}(1)-\mathrm{C}(9)$ & $2.06(2)$ \\
$\mathrm{Fe}(1)-\mathrm{C}(10)$ & $2.029(13)$ & $\mathrm{N}(1)-\mathrm{C}(44)$ & $1.55(2)$ & $\mathrm{N}(1)-\mathrm{C}(46)$ & $1.52(2)$ \\
$\mathrm{N}(1)-\mathrm{C}(47)$ & $1.50(2)$ & $\mathrm{N}(1)-\mathrm{C}(48)$ & $1.50(2)$ & $\mathrm{N}(2)-\mathrm{C}(32)$ & $1.57(2)$ \\
$\mathrm{N}(2)-\mathrm{C}(34)$ & $1.49(3)$ & $\mathrm{N}(2)-\mathrm{C}(35)$ & $1.54(2)$ & $\mathrm{N}(2)-\mathrm{C}(36)$ & $1.44(3)$ \\
$\mathrm{C}(4)-\mathrm{C}(31)$ & $1.49(2)$ & $\mathrm{C}(10)-\mathrm{C}(43)$ & $1.54(2)$ & $\mathrm{C}(31)-\mathrm{C}(32)$ & $1.57(2)$ \\
$\mathrm{C}(43)-\mathrm{C}(44)$ & $1.54(2)$ & $\mathrm{C}(31)-\mathrm{C}(37)$ & $1.53(2)$ & $\mathrm{C}(43)-\mathrm{C}(49)$ & $1.51(2)$ \\
$\mathrm{C}(32)-\mathrm{C}(33)$ & $1.54(3)$ & $\mathrm{C}(44)-\mathrm{C}(45)$ & $1.49(2)$ & & \\
$\mathrm{C}(4)-\mathrm{C}(31)-\mathrm{C}(32)$ & $106.7(12)$ & $\mathrm{C}(10)-\mathrm{C}(43)-\mathrm{C}(44)$ & $107.1(10)$ \\
$\mathrm{C}(4)-\mathrm{C}(31)-\mathrm{C}(37)$ & $113.5(11)$ & $\mathrm{C}(10)-\mathrm{C}(43)-\mathrm{C}(49)$ & $111.8(10)$ \\
$\mathrm{C}(32)-\mathrm{C}(31)-\mathrm{C}(37)$ & $117.5(12)$ & $\mathrm{C}(44)-\mathrm{C}(43)-\mathrm{C}(49)$ & $117.7(11)$ \\
$\mathrm{C}(31)-\mathrm{C}(32)-\mathrm{C}(33)$ & $113.8(14)$ & $\mathrm{C}(43)-\mathrm{C}(44)-\mathrm{C}(45)$ & $115.2(11)$ \\
$\mathrm{C}(31)-\mathrm{C}(32)-\mathrm{N}(2)$ & $112.7(14)$ & $\mathrm{C}(43)-\mathrm{C}(44)-\mathrm{N}(1)$ & $112.3(10)$ \\
$\mathrm{C}(33)-\mathrm{C}(32)-\mathrm{N}(2)$ & $114.3(14)$ & $\mathrm{C}(45)-\mathrm{C}(44)-\mathrm{N}(1)$ & $112.8(11)$ \\
\hline
\end{tabular}

Table I. Selected bond lengths $(\AA)$ and bond angles $\left({ }^{\circ}\right)$ of $\mathrm{Cp}_{2}{ }_{2} \mathrm{Fe} .2 \mathrm{MeI}$ (molecule 1).

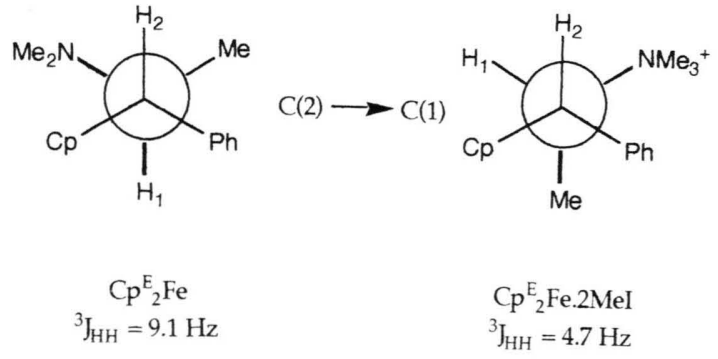

The gauche conformation of the hydrogens $\mathrm{H}(1)$ and $\mathrm{H}(2)$ around $\mathrm{C}(1)-\mathrm{C}(2)$ in $\mathrm{Cp}^{\mathrm{E}}{ }_{2} \mathrm{Fe} .2 \mathrm{MeI}$ seems to be maintained in solution. The ${ }^{1} \mathrm{H}$ NMR spectrum in $\mathrm{CD}_{3} \mathrm{OD}$ reveals a ${ }^{3} J_{\mathrm{HH}}$ coupling of $4.7 \mathrm{~Hz}$, which, through the Karplus equation, is in accord with the observed dihedral angle of $c a .60^{\circ}$. In contrast to this, the starting compound $\mathrm{Cp}_{2}{ }_{2} \mathrm{Fe}$ shows a ${ }^{3} J_{\mathrm{HH}}$ coupling of $9.1 \mathrm{~Hz}$, suggesting that $\mathrm{H}(1)$ and $\mathrm{H}(2)$ prefer an anti conformation. The reason for this difference remains as yet unclear. A further interesting spectral feature of $\mathrm{Cp}_{2}{ }_{2} \mathrm{Fe} .2-$ 
MeI is the ${ }^{2} J\left({ }^{13} \mathrm{C}^{-14} \mathrm{~N}\right)$ coupling of $3.4 \mathrm{~Hz}$ observed in the ${ }^{13} \mathrm{C}$ NMR spectrum.

Further progress in the coordination chemistry of the $\mathrm{Cp}^{\mathrm{E}}$ ligand is expected from the use of the nitrogen side arm for intramolecular coordination.

\section{Experimental}

The compounds listed below, although airstable, were synthesized under argon using carefully dried solvents. NMR spectra were measured on a Varian Gemini instrument operating at $300\left({ }^{1} \mathrm{H}\right)$ and 75.5 $\left({ }^{13} \mathrm{C}\right) \mathrm{MHz}$.

\section{$\mathrm{Cp}_{2}{ }_{2} \mathrm{Fe}$}

To an orange solution of $\mathrm{Cp}^{\mathrm{E}} \mathrm{Li}$ (prepared from $\mathrm{Cp}^{\mathrm{E}} \mathrm{H}(1.45 \mathrm{~g}, 6.4 \mathrm{mmol})$ and $n$-BuLi $(4.5 \mathrm{ml}$ of a $1.65 \mathrm{M}$ solution in hexane)) in $\mathrm{THF}(25 \mathrm{ml})$ was added $\mathrm{FeCl}_{2}(\mathrm{THF})_{1.5}(0.81 \mathrm{~g}, 3.4 \mathrm{mmol})$. The resulting suspension was stirred for $2 \mathrm{~d}$ at $60{ }^{\circ} \mathrm{C}$. Volatiles were removed in vacuo, and the residue extracted with petroleum ether. Concentrating and chilling to $-80{ }^{\circ} \mathrm{C}$ of the combined extracts afforded $\mathrm{Cp}^{\mathrm{E}}{ }_{2} \mathrm{Fe}$ as yellow-orange microcrystals $(1.0 \mathrm{~g}, 60 \%) .{ }^{1} \mathrm{H}$ NMR $\left(\mathrm{C}_{6} \mathrm{D}_{6}\right): \delta=0.66(3 \mathrm{H}, \mathrm{d}, 6.6$ $\left.\mathrm{Hz}, \mathrm{CHCH}_{3}\right), 2.09\left(6 \mathrm{H}, \mathrm{s}, \mathrm{N}\left(\mathrm{CH}_{3) 2}\right), 2.80(1 \mathrm{H}, \mathrm{dq}\right.$, $\left.\mathrm{CHCH}_{3}\right), 3.51(1 \mathrm{H}, \mathrm{d}, 9.1 \mathrm{~Hz}, \mathrm{CHPh}), 3.64,3.71$, $3.82,4.10\left(4 \mathrm{x} 1 \mathrm{H}, \mathrm{s}, \mathrm{C}_{5} H_{4}\right), 7.1-7.2\left(5 \mathrm{H}, \mathrm{m}, \mathrm{C}_{6} H_{5}\right)$. ${ }^{13} \mathrm{C}$ NMR $\left(\mathrm{C}_{6} \mathrm{D}_{6}\right): \delta=11.50\left(\mathrm{CHCH}_{3}\right), 40.91$ $\left(\mathrm{N}\left(\mathrm{CH}_{3}\right)_{2}\right), 50.26(\mathrm{CHPh}), 67.94\left(\mathrm{CHCH}_{3}\right), 64.74$, 67.35, 68.32, 70.23 (all $C_{5} \mathrm{H}_{4}$ ), 91.90 (ipso-C of Cp), 126.0, 127.86, 129.12, 144.26 (p,o, m, ipso-C of Ph).

Analysis for $\mathrm{C}_{32} \mathrm{H}_{40} \mathrm{FeN}_{2}$

$$
\begin{array}{llll}
\text { Calcd } & \text { C 75.6 } & \text { H 7.9 } & \text { N 5.5\%, } \\
\text { Found } & \text { C 74.7 } & \text { H } 8.7 & \text { N 5.1\%. }
\end{array}
$$

\section{$\mathrm{Cp}^{\mathrm{E}}{ }_{2} \mathrm{Fe} .2 \mathrm{MeI}$}

A solution of $\mathrm{Cp}^{\mathrm{E}}{ }_{2} \mathrm{Fe}$ in toluene or pentane when treated with an excess of MeI deposits a pale yellow powder within a few hrs at room temper- ature. Filtration and drying affords a quantitative yield of $\mathrm{Cp}^{\mathrm{E}}{ }_{2} \mathrm{Fe} .2 \mathrm{MeI} ; \mathrm{m}$. p. $177{ }^{\circ} \mathrm{C} .{ }^{1} \mathrm{H}$ NMR $\left(\mathrm{CD}_{3} \mathrm{OD}\right): \delta=0.65\left(3 \mathrm{H}, \mathrm{d}, 6.5 \mathrm{~Hz}, \mathrm{CHCH}_{3}\right), 3.06$ $\left(9 \mathrm{H}, \mathrm{s}, \mathrm{N}\left(\mathrm{CH}_{3) 3}\right), 3.94\left(1 \mathrm{H}, \mathrm{dq}, \mathrm{CHCH}_{3}\right), 3.62\right.$, $3.78,3.87,4.62\left(4 \times 1 \mathrm{H}, \mathrm{s}, \mathrm{C}_{5} H_{4}\right), 4.78(1 \mathrm{H}, \mathrm{d}, 4.7 \mathrm{~Hz}$, $\mathrm{CHPh}), \quad 6.7-6.9\left(5 \mathrm{H}, \quad \mathrm{m}, \mathrm{C}_{6} H_{5}\right) .{ }^{13} \mathrm{C}$ NMR $\left(\mathrm{CD}_{3} \mathrm{OD}\right): \delta=11.99\left(\mathrm{CHCH}_{3}\right), 47.78(\mathrm{CHPh})$, $53.67\left(\mathrm{t}, 3.4 \mathrm{~Hz}, \mathrm{~N}\left(\mathrm{CH}_{3}\right)_{3}\right), 77.69\left(\mathrm{CHCH}_{3}\right), 70.10$, 71.16, 71.23, $72.24\left(\right.$ all $\left.C_{5} \mathrm{H}_{4}\right), 91.72$ (ipso- $C$ of $C \mathrm{p}$ ), 129.47, 130.56, 131.62, 140.58 ( $p, o, m$, ipso- $C$ of $\mathrm{Ph})$.

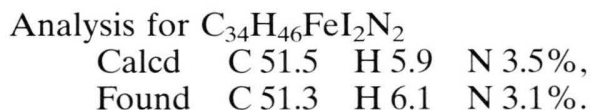

Crystals suitable for X-ray diffraction work were grown by slow diffusion of diethyl ether into a solution of the iron compound in methanol over a period of weeks.

\section{Crystal structure determination of $\mathrm{CP}_{2}{ }_{2} \mathrm{Fe} .2 \mathrm{MeI}$.}

Siemens SMART-diffractometer. Mo- $\mathrm{K}_{\alpha}$ radiation. Structure solution with SHELXS-86 [5], refinement with SHELXL-93 [6]. Full-matrix leastsquares refinement on $\mathrm{F}^{2} . \mathrm{C}_{34} \mathrm{H}_{46} \mathrm{FeI}_{2} \mathrm{~N}_{2}$ : triclinic space group P1, $a=14.095(4), b=15.374(5), c=$ 16.243(5) A, $\alpha=63.772(5), \beta=64.329(4), \gamma=$ 71.827(4) ${ }^{\mathrm{o}}, \mathrm{V}=2814.1(14) \AA^{3}, \mathrm{Z}=3, \mathrm{~d}_{\text {calcd }}=1.403$ $\mathrm{g} \mathrm{cm}^{-3}, \mu=2.069 \mathrm{~mm}^{-1}, \mathrm{~F}(000)=1188$. Reflections collected at $293 \mathrm{~K}: 13114$ (full index range with 1.5 $<\theta<23.3^{\circ}$ ) of which 10829 with $\mathrm{I}>2 \sigma$ (I) were used. Absorption correction was applied. The nonhydrogen atoms were refined anisotropically. Final $R_{1}=0.0515, w R_{2}=0.1409(\mathrm{I}>2 \sigma) ; R_{1}=0.0600$, $w R_{2}=0.1532$ (all data); absolute structure parameter 0.03(3).

\section{Acknowledgement}

The 'Deutsche Forschungsgemeinschaft' is thanked for financial support. Prof. Dr. K. H. Thiele is thanked for his continuous support and interest. 
[1] Some recent papers: a) K. Jonas, P. Klusmann, R. Goddard, Z. Naturforsch. 50b, 394 (1995);

b) Y. Mu, W. E. Piers, L. R. MacGillivray, M. J. Zaworotko, Polyhedron 14, 1 (1995);

c) M. D. Fryzuk, S. S. H. Mao, P. B. Duval, S. J. Rettig, Polyhedron 14, 11 (1995);

d) A. H. Cowley, C. S. King, A. Decken, Organometallics 14, 20 (1995);

e) P. Van de Weghe, C. Bied, J. Collin, J. Marcalo, I. Santos, J. Organomet. Chem. 475, 121 (1994);

f) W. A. Herrmann, M. J. A. Morawietz, J. Organomet. Chem. 482, 169 (1994);

g) W. A. Herrmann, M. J. A. Morawietz, T. Priermeier, Angew. Chem. 106, 2025 (1994);

h) I. Lee, F. Dahan, A. Maisonnat, R. Poilblanc, Organometallics 13, 2743 (1994);

i) Y. Qian, J. Huang, X. Chen, G. Li, W. Chen, B. Li, X. Jin, Q. Yang, Polyhedron 11, 1105 (1994);

j) S. Zhang, X. Zhuang, G. Wei, W. Chen, Polyhedron 13, 2867 (1994):

k) D. Deng, X. Zheng, C. Qian, J. Sun, L. Zhang, J. Organomet. Chem. 466, 95 (1994);

1) C. Qian, X. Zheng, B. Wang, D. Deng, J. Sun, J. Organomet. Chem. 466, 101 (1994);

m) C. Qian, D. Zhu, J. Chem. Soc., Dalton Trans. 1599 (1994);

n) G. Paolucci, R. D'Ippolito, C. Ye, C. Qian, J. Gräper, D. R. Fischer, J. Organomet. Chem. 471, 97 (1994);

o) D. Deng, X. Zheng, C. Qian, J. Sun, A. Dormond, D. Baudry, M. Visseaux, J. Chem. Soc., Dalton Trans. 1665 (1994);

p) D. Deng, Y. Jiang, C. Qian, G. Wu, P. Zheng, J. Organomet. Chem. 470, 99 (1994);

q) C. Qian, B. Wang, D. Deng, J. Hu, J. Chen, G. Wu, P. Zheng, Inorg. Chem. 33, 3382 (1994).

[2] a) T.-F. Wang, T.-Y. Lee, J.-W. Chou, C.-W. Ong, J. Organomet. Chem. 423, 31 (1992);

b) T.-F. Wang, Y.-S. Wen, J. Organomet. Chem. 439 , 155 (1992);

c) P. Jutzi, J. Dahlhaus, M. O. Kristen, J. Organomet. Chem. 450, C1(1993);

d) P. Jutzi, J. Dahlhaus, M. Bangel, J. Organomet. Chem. 460, C13 (1993); e) J. Dahlhaus, M. Bangel, P. Jutzi, J. Organomet. Chem. 474, 55 (1994);

f) P. Jutzi, M. Bangel, J. Organomet. Chem. 480, C18 (1994);

g) P. Jutzi, J. Dahlhaus, Coord. Chem. Rev. 137, 179 (1994);

h) P. Jutzi, J. Kleimeier, J. Organomet. Chem. 486, 287 (1995);

i) W. A. Herrmann, R. Anwander, F. C. Munck, W. Scherer, Chem. Ber. 126, 331 (1993);

j) R. Anwander, W. A. Herrmann, W. Scherer, F. C. Munck, J. Organomet. Chem. 462, 163 (1993);

k) W. S. Rees, K. A. Dippel, Org. Prep. Proc. Int. 24, 527 (1992);

1) W. S. Rees, U. W. Lay, K. A. Dippel, J. Organomet. Chem. 483, 27 (1994);

m) J. C. Flores, J. C. W. Chien, M. D. Rausch, Organometallics 13, 4140 (1994).

[3] A. A. H. van der Zeijden, Tetrahedron: Asymmetry 6, 913 (1995). The first enantiomerically pure Cp ligand bearing a nitrogen donor atom was recently reported: H. Adams, N. A. Bailey, M. Colley, P. A. Schofield, C. White, J. Chem. Soc., Dalton Trans. 1994, 1445.

[4[ a) M. C. Grossel, M. R. Goldspink, J. A. Hriljac, S. C. Weston, Organometallics 10, 851 (1991); b) G. Ferguson, J. F. Gallagher, C. Glidewell, C. M. Zakaria, Acta Crystallogr. C49, 967 (1993); c) J. F. Gallagher, G. Ferguson, C. Glidewell, C. M. Zakaria, Acta Crystallogr. C50, 18 (1994);

d) G. Ferguson, J. F. Gallagher, C. Glidewell, C. M. Zakaria, Acta Crystallogr. B50, 146 (1994).

[5] G. M. Sheldrick, SHELXS-86, Program for the solution of crystal structures, Göttingen (1986).

[6] G. M. Sheldrick, SHELXL-93, Program for the refinement of crystal structures, Götingen (1993).

[7] Further details of the crystal structure determination may be obtained from the Fachinformationszentrum Karlsruhe, Gesellschaft für wissenschaftlich-technische Information mbH, D-76344 Eggenstein-Leopoldshafen, on quoting the depository number CSD 404337, the names of the authors and the journal citation. 